\title{
Dynamical screening function and plasmons in the wide HgTe quantum wells at high temperatures
}

\author{
E.O. Melezhik ${ }^{1, *}$, F.F. Sizov ${ }^{1}$, N.N. Mikhailov², J.V. Gumenjuk-Sichevska ${ }^{1}$ \\ ${ }^{I}$ V. Lashkaryov Institute of Semiconductor Physics NAS of Ukraine, Kyiv, Ukraine \\ ${ }^{2}$ Institute of Semiconductor Physics SB of RAS, Novosibirsk 630090, Russia \\ *E-mail: emelezhik@gmail.com
}

\begin{abstract}
The dynamical screening function of two-dimensional electron gas in a wide HgTe quantum well (QW) has been numerically modeled in this work. Calculations were performed in the Random Phase Approximation (RPA) framework and were based on the Lindhard equation. Our simulations directly incorporated non-parabolicity of bulk 2D carriers' spectrum, which was obtained by full 8-band k.p method. Known from literature are the data that transport properties of $\mathrm{HgTe}$ QWs can be explained by graphene-like screening. We carried out the comparison of the screening function for the Schrödinger fermions in the inverted bands HgTe QW with the appropriate screening function for graphene monolayer with the Dirac fermions. In addition, the dependences of HgTespecific screening function on temperature, scattering wave-vector and frequency have been studied with the purpose to ascertain the transport properties under high frequency radiation for the QW structures to be used as THz detectors. Plasmon frequencies of 2DEG in HgTe quantum well under study were calculated in the long-wave limit for $T=77 \mathrm{~K}$.
\end{abstract}

Keywords: dielectric function, 2DEG, HgTe, quantum wells, Lindhard equation, Random Phase Approximation.

doi: https://doi.org/10.15407/spqeo21.02.187

PACS 71.45.Gm, 73.21.Fg, 77.22.Ch

Manuscript received 27.02.18; revised version received 26.04.18; accepted for publication 27.06.18; published online 03.07.18.

\section{Introduction}

The development of novel detectors operating in the terahertz $(\mathrm{THz})$ spectral range is one of the important challenges of modern optoelectronics.

HgTe quantum wells (QWs) with the width larger than $\sim 6.7 \mathrm{~nm}$ have inverted bands order inside the well, and therefore semimetal conductivity type seems attractive for $\mathrm{THz}$ signal detection because the QWs can be characterized by high electron mobility and high electron concentrations even at liquid nitrogen temperatures [5]. The electron mobility order of $10^{5} \mathrm{~cm}^{2} / \mathrm{V} \cdot \mathrm{s}$ was experimentally reached in such structures at $T=77 \mathrm{~K}[14,15]$, while theoretical estimations predict that this mobility can be increased up to $(6 \ldots 8) \cdot 10^{5} \mathrm{~cm}^{2} / \mathrm{V} \cdot \mathrm{s}$. Moreover, they have much lower resistivity and lower thermal noise in comparison with the direct bands order $\mathrm{HgCdTe}$ QWs [1]. All these features of $\mathrm{HgTe}$ quantum wells with the inverted bands order make them rather important for various applications in the field of $\mathrm{THz}$ detection at the moderate cooling regime. In this paper, we deal with $\mathrm{HgTe}$ QWs with inverted bands order at the temperature $77 \mathrm{~K}$, at which these structures can be exploited as $\mathrm{THz}$ detectors.

The phenomena of Coulomb charge screening of carrier scattering by two-dimensional electron gas (2DEG) has the key importance for theoretical study of high frequency transport and radiation absorption in materials with the high electron concentration. The form of dielectric screening function sufficiently relies on the energy dispersion law for two-dimensional carriers. Due to strong nonparabolicity of such a dispersion law in HgTe QWs, the dielectric function for two-dimensional systems with parabolic dispersion cannot be applied to our case. There exists experimental evidence that the dielectric screening function for $\mathrm{HgTe}$ QWs with the stabilized surface state transport in topological insulator phase is close to the dielectric function of graphene with the linear energy dispersion law [2]. In contrast to the case of Dirac fermions, there does not exist any analytical form for dielectric screening function of $2 \mathrm{DEG}$ in HgTe inverted bands QWs for the Schrödinger fermions. This case occurs when the temperature is high enough to cancel edge states transport, or doping level is 
far from the intrinsic case, and electron states are heavily degenerated. This case also differs from the parabolic dispersion case due to a strong band mixing and high density of states. However, to create detectors operating with moderate cooling, this case is topical. Thus, knowledge of dielectric screening function for the inverted bands $\mathrm{HgTe} \mathrm{QW}$ with its unique dispersion law is of key importance for further modeling of any transport processes in it.

In the literature, there exist calculations of dynamical screening function for $\mathrm{HgTe}$ quantum wells near the phase transition [12]. However, this model based on the four-band calculations of energy spectrum is applicable only at the liquid helium temperatures and cannot be used even at $77 \mathrm{~K}$.

Current work dedicates to the numerical modeling of dielectric screening function and plasmon frequencies for HgTe QWs in Random Phase Approximation (RPA) formalism, which was previously successfully applied to the modeling of such function in graphene [3]. In the RPA, electrons respond only to the total electric potential that is consisting of an external perturbing potential and a screening potential. In this assumption, the perturbing potential oscillates at a frequency of external radiation source $w$, so that the model gives self-consistent dynamic dielectric function, which is called the Lindhard dielectric function.

In this work all simulations were provided for $\mathrm{Hg}_{0.15} \mathrm{Cd}_{0.85} \mathrm{Te} / \mathrm{HgTe} / \mathrm{Hg}_{0.15} \mathrm{Cd}_{0.85} \mathrm{Te}$ quantum well of $20 \mathrm{~nm}$ width, at the moderate cooling temperature, for arbitrary values of scattering wave-vector $q$ and frequency $w$. Quantum well had two $n$-type delta-doped layers in the barriers, at the distance of $10 \mathrm{~nm}$ from QW interfaces. Width of delta-doped layers was $15 \mathrm{~nm}$, concentration of dopant was $10^{17} \mathrm{~cm}^{-3}$. We assume that all free electrons from the delta-doped layers are injected into the well.

\section{Dielectric screening function in RPA formalism}

\section{A. Lindhard equation}

Numerical simulation of HgTe QW dielectric screening function is based on the well-known Lindhard equation for dynamical dielectric function:

$\varepsilon(\vec{q}, w)=1-V_{q} \sum_{\vec{k}} \frac{f_{\vec{k}-\vec{q}}-f_{\vec{k}}}{\hbar(w+i s)+E_{\vec{k}-\vec{q}}-E_{\vec{k}}}$.

Here, $f_{k}$ is the Fermi-Dirac distribution function, $E_{k}-$ energy dispersion law for the given system, $s$ - positive infinitesimal that is taken in the limit of going to +0 to produce the real part, $V_{q}$ - Fourier transform of the Coulomb energy for 2DEG in vacuum. The expression for $V_{q}$ is as follows [3]:
$V_{q}=\left(2 \pi e^{2} / \kappa q\right) F(q)$

where $\kappa$ is the dielectric constant of the lattice. The formfactor $F(q)$ will be taken equal to 1 , which corresponds to the strict two-dimensional limit [4].

Suggesting the case, when only ground level of quantum well is populated, and for two-dimensional medium, the last equation can be rewritten as:

$\varepsilon(\vec{q}, w)=1-\frac{e^{2}}{\pi \kappa q} \iint d^{2} \vec{k} \frac{f_{\vec{k}-\vec{q}}-f_{\vec{k}}}{\hbar(w+i s)+E_{\vec{k}-\vec{q}}-E_{\vec{k}}}$,

where we have substituted the value for $V_{q}$ and converted the sum over all occupied electron states to the integral over 2D electron wave-vector $\vec{k}$.

Using the Dirac identity:

$\lim _{s \rightarrow 0} \frac{1}{X+i s}=p \cdot v \cdot \frac{1}{X}-i \pi \delta(X)$,

where p.v. denotes the principal value of an integral and $\delta(X)$ is the Dirac delta function, we can obtain the real and imaginary parts of the dielectric function.

\section{B. Real and imaginary parts of dielectric function}

Using the polar coordinates and neglecting the dependence of electron energy on orientation of its wavevector, we can write real and imaginary parts of dielectric function as:

$$
\begin{gathered}
\varepsilon_{\mathrm{RE}}(\vec{q}, w)=1-\frac{e^{2}}{\pi \kappa q} \iint d k d \theta \cdot k \frac{f_{|\vec{k}-\vec{q}|}-f_{\vec{k}}}{\hbar w+E|\vec{k}-\vec{q}|-E_{\vec{k}}}, \\
\varepsilon_{\mathrm{IM}}(\vec{q}, w)=-2 \frac{e^{2}}{\kappa q} \int d k \cdot k \frac{\left(f_{|\vec{k}+\vec{q}|}-f_{\vec{k}}\right)|\vec{k}-\vec{q}|}{\left|\frac{\partial E}{\partial k}\right|_{|\vec{k}+\vec{q}|} \cdot k \cdot q \cdot \sin \theta_{0}},
\end{gathered}
$$

where $\theta_{0}$ is defined as a root of the equation $E_{\vec{k}+\vec{q}}-E_{\vec{k}}-\hbar w=0$ at given values of $k$ and $q$. If at the given point of integration mesh no real $\theta_{0}$ exists, the integrand of the last equation at this point is considered to be zero, because the Delta-function argument at this point will be nonzero.

From the equations for $\varepsilon_{\mathrm{RE}}$ and $\varepsilon_{\mathrm{IM}}$ (5), it follows that for the case of static screening $(w=0), \varepsilon_{\mathrm{IM}}(q, w)=0$, 
because from the requirement of $E_{\vec{k}+\vec{q}}-E_{\vec{k}}=0$ it follows that $f_{\vec{k}+\vec{q}}-f_{\vec{k}}=0$.

As the dispersion law $E_{k}$ for $\mathrm{HgTe}$ quantum wells can be calculated only numerically in 8-band Kane formalism, no analytic equations for $\varepsilon_{\mathrm{RE}}(q, w)$ and $\varepsilon_{\mathrm{IM}}(q, w)$ are possible. Calculations of real and imaginary parts of dielectric function should be done numerically being based on the particular form of dispersion equation.

\section{Energy spectra of HgTe quantum wells}

Calculations of energy spectra are performed in the framework of the envelope functions approach, when the carrier wave-function is expanded on the basis of eight Bloch band-edge (in-plane $k=0$ ) functions [5]. The system is assumed to be periodical, although the barrier well width is chosen to correspond to the isolated quantum well. Our calculations were made for the quantum well in (100) crystal plane, thus $z$-axis coincides with the growth direction of the well. Energy levels and envelope-function coefficients were found from the simultaneous solution of the system of coupled differential equations - Hamiltonian equation and boundary conditions system [5].

The calculated energy spectrum of localized carriers in $\mathrm{Hg}_{0.15} \mathrm{Cd}_{0.85} \mathrm{Te} / \mathrm{HgTe} / \mathrm{Hg}_{0.15} \mathrm{Cd}_{0.85} \mathrm{Te}$ quantum well at the temperature $77 \mathrm{~K}$ is shown in Fig. 1. One can see that inverted bands order is formed inside the well, because the band gap between electron and hole levels is absent. The small gap between ground electrons and ground holes' levels is explained by the presence of mismatch strain and quantization of energy spectrum in twodimensional medium.

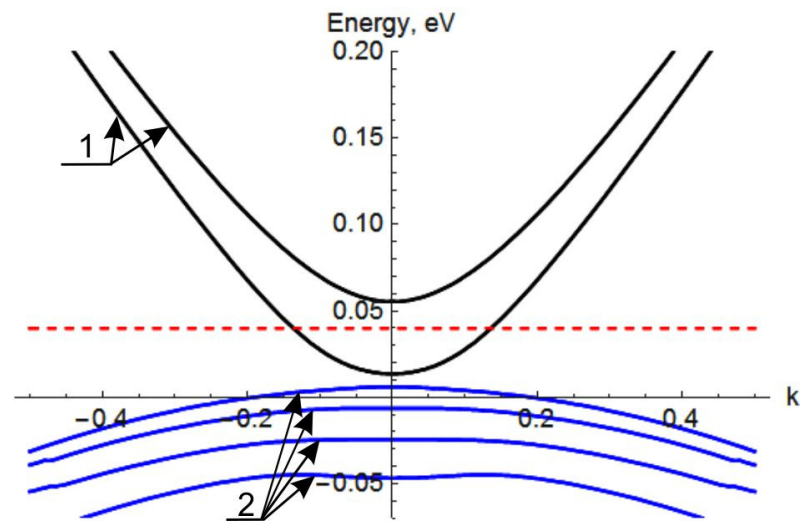

Fig. 1. Energy spectrum of localized carriers in $\mathrm{Hg}_{0.15} \mathrm{Cd}_{0.85} \mathrm{Te} / \mathrm{HgTe} / \mathrm{Hg}_{0.15} \mathrm{Cd}_{0.85} \mathrm{Te}$ quantum well at $T=77 \mathrm{~K}$. Dashed line represents the position of Fermi level in the considered sample. The horizontal axis represents twodimensional wave-vector $k$ in the units of $\mathrm{nm}^{-1}$. Black curves (1) represent the conduction band, while blue curves (2) represent the valence band.
Due to the proximity of electron and heavy hole bands as well as doping the barriers, the Fermi level is shifted high into the conduction band. The distance between the Fermi level and the bottom of first electron level is greater than $2 k_{\mathrm{B}} T$. Also, the distance from the Fermi level to the top of ground heavy holes level is about $5 k_{\mathrm{B}} T$. Thus, for this sample we can suggest that only ground level electrons take part in the screening processes.

Electron levels in Fig. 1 are close to parabolic in the center of the zone, while they become almost linear at high values of wave-vector $\vec{k}$. Thus, this system cannot be considered in parabolic approximation (as classical semiconductors).

There exists the experimental evidence that transport properties of two-dimensional HgTe layers can be explained by graphene-like type of screening of 2DEG [2]. Thus, comparison of graphene and HgTe QW specific dielectric functions of $2 \mathrm{DEG}$ and discussion about their qualitative differences are one of the key points of this study.

\section{Results and discussion}

In a general case, the dielectric function of 2DEG depends on scattering wave-vector $q$ and frequency $w$. However, such scattering mechanisms as charged impurities scattering and electron-hole scattering are described by static screening (when frequency $w=0$ ).

Three static screening functions - for QW with parabolic dispersion, for graphene and for $\mathrm{HgTe} \mathrm{QW}-$ are compared in Fig. 2 for the temperature $77 \mathrm{~K}$. One should note that in the long-wave limit, when the scattering wave-vector $q$ goes to zero $(q \rightarrow 0)$, all three functions coincide. However, at large values of the

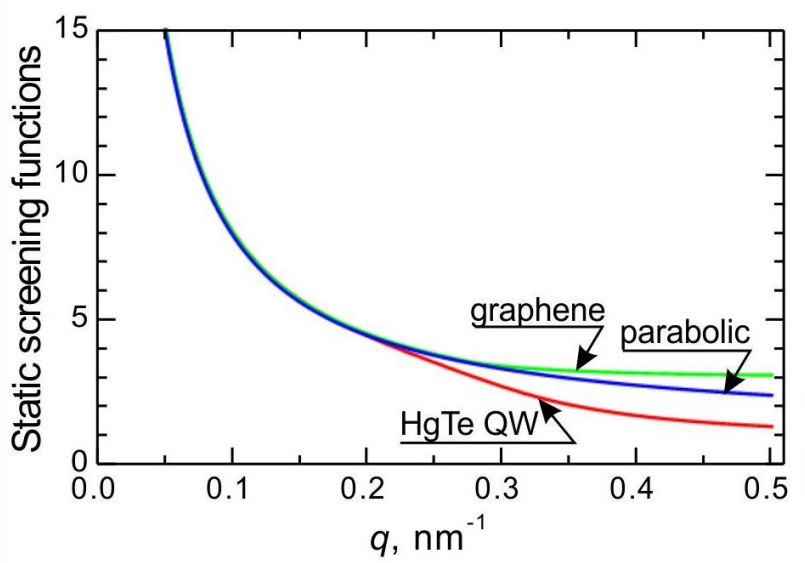

Fig. 2. Comparison of static screening functions for the graphene monolayer, QW of the thickness $20 \mathrm{~nm}$ with parabolic dispersion and HgTe QW of the same thickness at $T=77 \mathrm{~K}$. The horizontal axis represents the two-dimensional wave-vector $q$ in the units of $\mathrm{nm}^{-1}$. 


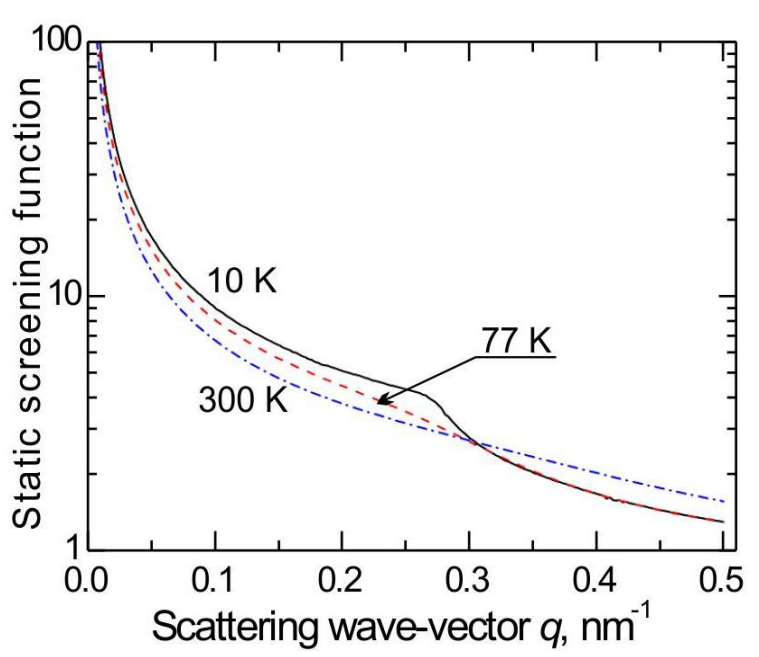

Fig. 3. Static screening functions for $\mathrm{HgTe}$ QW, calculated at three different temperatures: 10,77 , and $300 \mathrm{~K}$. The horizontal axis represents the two-dimensional wave-vector $q$.

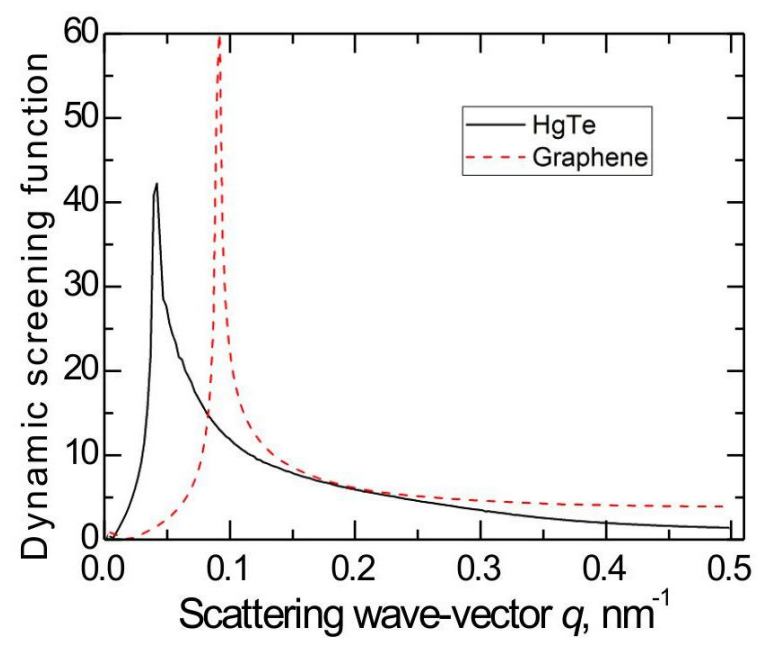

Fig. 4. Dynamic dielectric functions for $\mathrm{HgTe}$ quantum well and graphene. Calculations were performed for the frequency of longitudinal optical phonon with the energy $17 \mathrm{meV}$ at $T=77$ K. The horizontal axis represents the two-dimensional scattering wave-vector $q$ in the units of $\mathrm{nm}^{-1}$.

scattering wave-vector $q$, the dielectric function for $\mathrm{HgTe}$ quantum well is several times smaller than the graphene one, which causes smaller damping of large-angle scattering on charged centers.

To make a comparison between screening processes in HgTe QW states and graphene Dirac states, all static and dynamic screening functions for graphene represented in the following Figures were calculated using methods and formulae from [3], while substituting the Fermi level and actual density of states at this level from the actual HgTe QW sample.

Similar dependences of static screening function for 20-nm width HgTe quantum well for three different temperatures $(10,77$, and $300 \mathrm{~K})$ are presented in Fig. 3. One can see that in the long-wave limit (when scattering wave-vector $q$ approaches zero), screening functions for all three temperatures coincide. Growth of $q$ leads to the decrease of the screening functions for all temperatures. It is interesting to note the appearance of bending at the curve for $T=10 \mathrm{~K}$ in Fig. 3 . The value of Fermi wavevector for this temperature is $0.14 \mathrm{~nm}^{-1}$. Discussed bending is located near $q=2 k_{\mathrm{F}}$ value, while its sharpness is decreased with the temperature growth.

Dynamic screening functions for $\mathrm{HgTe} \mathrm{QW}$ and graphene, at the frequency of optical phonon with the energy $17 \mathrm{meV}$ (LO frequency), are presented in Fig. 4. It is interesting to note that transition from purely linear graphene spectrum to nonparabolic HgTe spectrum leads to the removal of singularity point from the graph and increase of the peak width. In addition, the position of peak shifts to smaller values of $q$. Thus, $\mathrm{HgTe} \mathrm{QW}$ shows stronger screening for the scattering processes of electron by optical phonons as compared to the graphene dielectric function.

Another interesting point is the dependence of dynamic HgTe QW screening function on the temperature. Dependences of this function on the scattering wave-vector at LO frequency, for three different temperatures, have been plotted in Fig. 5. One can see that the increase of temperature shifts the position of maximum to higher values of $q$, at the same time decreasing its height. Nevertheless, the form of the dependence of screening function on $q$ in general remains the same. Consequently, the heating of electron, which takes place for example in $\mathrm{THz}$ hot-electron bolometers, should not result in sufficient changes of this 2DEG screening phenomenon. The nature of furcation of the peak for $T=10 \mathrm{~K}$ is not clear now however the increase of calculation accuracy does not lead to its disappearance.

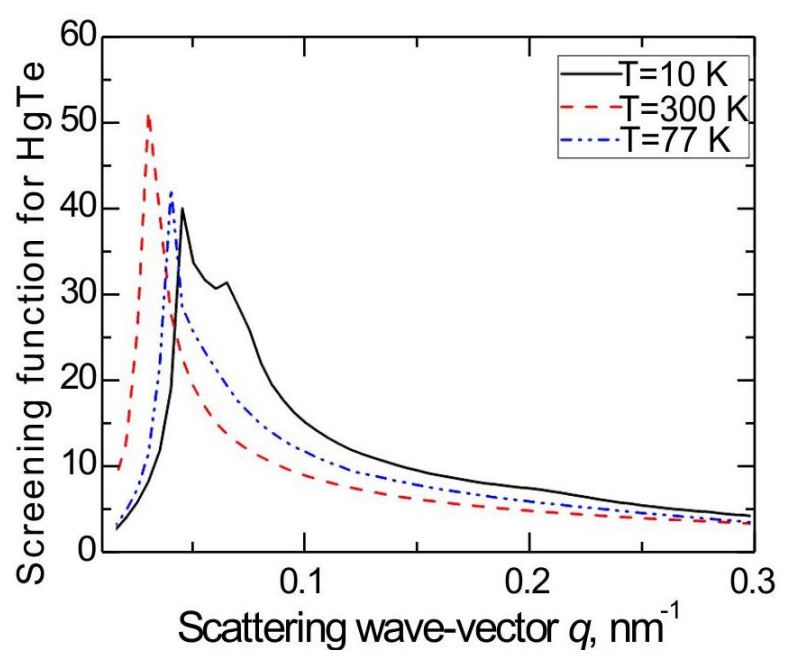

Fig. 5. Dynamic screening function for $\mathrm{HgTe} \mathrm{QW}$ at the frequency of longitudinal optical phonon with the energy $17 \mathrm{meV}$ at $T=10 \mathrm{~K}, 77 \mathrm{~K}$ and $300 \mathrm{~K}$. The horizontal axis represents the two-dimensional wave-vector $q$ in the units of $\mathrm{nm}^{-1}$. 


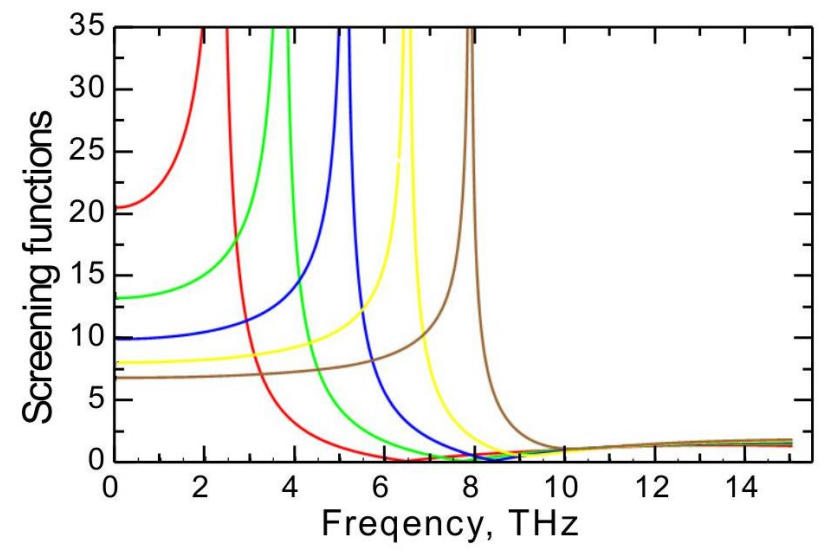

Fig. 6. Linear frequency dependences of screening function for graphene. The horizontal axis represents the frequency in THz. Each of the curves is built for the fixed value of scattering wave-vector $q$. From the left to the right, appropriate scattering wave-vectors of these curves are $0.05,0.08,0.11,0.14$ and $0.17 \mathrm{~nm}^{-1}$.

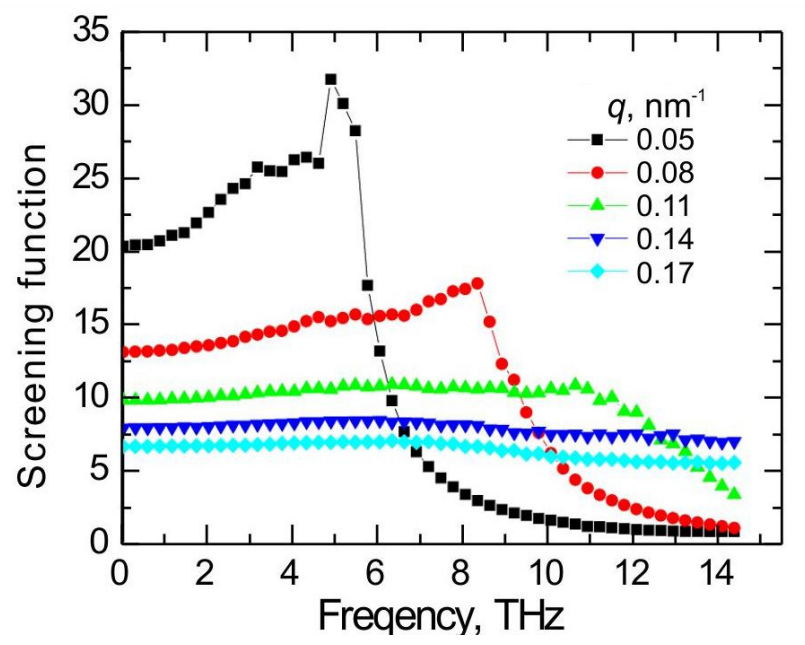

Fig. 7. Linear frequency dependences of screening function for HgTe quantum well. The horizontal axis represents the frequency in THz. Each of the curves is built for the fixed value of scattering wave-vector $q$. From the higher to the lower curves, appropriate scattering wave-vectors of these curves are $0.05,0.08,0.11,0.14$ and $0.17 \mathrm{~nm}^{-1}$.

In the final part of the article, we present the results for frequency dependences of $\mathrm{HgTe} \mathrm{QW}$ and graphene screening functions.

Fig. 6 represents frequency dependences of graphene screening function for different values of scattering wave-vector $q$. Each curve has sharp maximum, while small minimum emerges to the right of the peak. At high frequencies, all curves become constant and coinciding between each other.

Fig. 7 represents appropriate frequency dependences of screening functions for $\mathrm{HgTe}$ quantum well. Small spikes in the curves could be explained by the computational errors. Comparing the data from Fig. 7 with those from Fig. 6, one can note several important differences.

Transition from purely linear (graphene) spectrum to nonparabolic (HgTe QW) spectrum leads to the removal of narrow peaks with an infinite height. They are replaced by wide plateaus, which height is decreased during the increase of scattering wave-vector $q$, while the width of these plateaus is sufficiently broader than that of corresponding peaks in Fig. 4. As one can see in these two highest curves (which correspond to the lowest values of $q$ ), maximum value frequencies of $\mathrm{HgTe} \mathrm{QW}$ screening function are higher than the corresponding frequencies from Fig. 6.

From the comparison of curves in Figs. 6 and 7 corresponding to the same value of scattering wavevector, we can make several conclusions.

For the frequencies that are higher than the peak value frequency, 2DEG screening in $\mathrm{HgTe}$ QW is much more efficient than in the graphene case. On the other hand, no frequencies do exist in $\mathrm{HgTe} \mathrm{QW}$, for which absolute screening (e.g., infinitely large screening function) of $2 \mathrm{DEG}$ is possible.

\section{Plasmons in RPA}

\section{A. Problem overview}

Two-dimensional plasmons or collective oscillations of the density of 2DEG promote absorption of incoming radiation at the frequencies close to $1 \mathrm{THz}$. This feature makes them promising for design of $\mathrm{THz}$ detectors. As inverted bands $\mathrm{HgTe}$ quantum wells can provide high electron mobility, low channel resistance and noise, calculation of their plasma frequencies is quite important.

In literature, they usually model the situation when only interface states are used for the carrier transport [6]. In the case of topological insulator, the interface levels of QW lie in the band gap of the energy spectrum of "bulk" 2D carriers. These interface levels are characterized by Dirac-like energy dispersion originating from the sign reversal of carrier mass at the interface. However, such interface plasmons are essential only when transport via bulk levels of QW is suppressed, which means that the Fermi level is in the middle of QW band gap, while this band gap is much higher than $k_{\mathrm{B}} T$. It results in the usage of very low temperatures [7] or heavily strained $\mathrm{HgTe}$ QW samples with wide band gap (up to $200 \mathrm{meV}$ ) [8]. Both approaches are undesirable for construction of real devices, because deep cooling results in bulky and expensive devices, while heavily strained samples could be affected by fast degradation.

Also, in literature, there exist numerical calculations describing plasmons in the bulk levels of HgTe quantum well $[9,10]$. However, they are made in the parabolic approximation, neglecting bands hybridization and nonparabolicity of energy spectrum of bulk carriers. Moreover, in [9] they are performed assuming that $T=0$. 
In [10], temperature is not mentioned in the text or in formulae, thus one could suggest that this work is also carried out for $T=0$.

Thus, up to our knowledge, no numerical simulations of frequency dispersion of "bulk" plasmons (existing at bulk levels of $\mathrm{HgTe} \mathrm{QW}$ ) can be found in literature for $T=77 \mathrm{~K}$.

Nevertheless, there exist experiments that allow one to suggest real existence of such plasmons in $\mathrm{HgTe}$ QW at liquid nitrogen and higher temperatures [11].

Presented in the following subsection are our numerical results for the frequency dispersion of "bulk" plasmons in $\mathrm{HgTe} \mathrm{QW}$ at $77 \mathrm{~K}$. These results properly incorporate bands mixing and non-parabolicity of dispersion law.

\section{B. Our numerical results for plasmons in RPA}

Calculation of screening function in the RPA framework has the immediate theoretical consequence for plasmonic frequencies. Due to physics incorporated in RPA, zero values of dielectric screening function (1) correspond to the collective excitations of 2DEG. Thus, calculation of frequencies and scattering wave-vectors, at which the screening function is zero, gives us the plasmon dispersion spectrum of QW. We will carry out these calculations in the long-wave limit $(q \rightarrow 0)$.

In this limit, the real part of screening function (5) can be rewritten as:

$$
\varepsilon_{\mathrm{RE}}^{\text {long-wave }}(\vec{q}, w)=1+\frac{e^{2}}{\varepsilon_{0} q \pi} \iint \frac{d k \cdot d \theta \cdot k \cdot q \cdot \cos \theta \frac{d f_{k}}{d k}}{\hbar w-q \cdot \cos \theta \frac{d E_{k}}{d k}}
$$

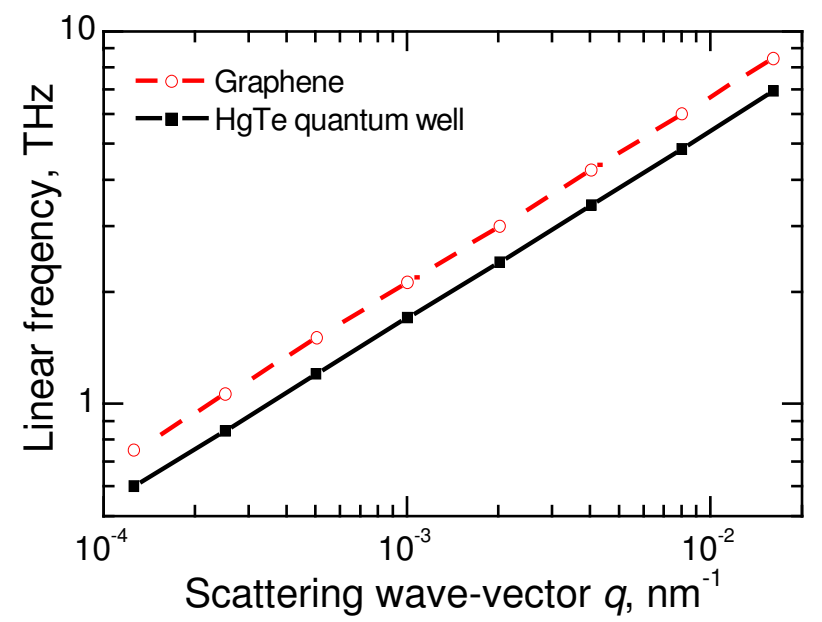

Fig. 8. Plasmon frequencies (in $\mathrm{THz}$ ) for $\mathrm{HgTe} \mathrm{QW}$ and graphene, in the dependence on the scattering wave-vector $q$. Plasmon frequencies for $\mathrm{HgTe} \mathrm{QW}$ were calculated for $T=$ $77 \mathrm{~K}$. Plasmon frequencies for graphene were calculated for $T=$ $0 \mathrm{~K}$ using the formula (18) from [3].
The imaginary part of screening function (5) is calculated via one-dimensional integral, thus it does not need any further simplification, as its integration error is already much smaller than for the real part.

Plasmon frequencies for $\mathrm{HgTe} \mathrm{QW}$ and single-layer graphene are presented in Fig. 8. Both calculations are carried out in the long-wave limit. Calculations for $\mathrm{HgTe}$ QW were performed using Eqs. (5) and (6) at $T=77 \mathrm{~K}$. Calculations for graphene were performed using the formula (18) from [3] at $T=0 \mathrm{~K}$. One can see that both dependences are presented as parallel straight lines in the double-logarithmic scale.

Plasmons following from our RPA calculations are not interface plasmons, because they are based on the $2 \mathrm{D}$ wavefunction of electrons localized inside the well. As our RPA calculations are carried out for temperatures much higher than the liquid helium temperature, appropriate plasmons are referred in this work as "hightemperature" plasmons.

Another important difference of high-temperature plasmons considered in this work and low-temperature interface plasmons from the literature lies in the coupling of electron spin and electron motion direction. In this case, near the phase transition point four-band Dirac Hamiltonian describes the energy spectrum of $\mathrm{HgTe}$ QW at the liquid helium temperature [13]. Such description allows one to present Hamiltonian in the form when its right-lower part is the complex conjugate of its left-upper part, while left-lower and right-upper parts of Hamiltonian being zero. From this form of Hamiltonian, one can note the presence of time-reversal symmetry of the system. It means that the direction of electron motion and the direction of its spin are coupled (in the absence of magnetic field and magnetic impurities), plasma waves are accompanied by spin waves, and the total phenomenon is called spin-plasmons.

By contrast, even at the liquid nitrogen temperature and far from the phase transition point, the energy spectrum cannot be modeled in 4-band approximation. 8-band k.p "high-temperature" plasmons calculations of the energy spectrum do not allow presenting Hamiltonian in the "time-reversal" form. This means that in the general case, direction of electron motion and direction of electron spin are not coupled, while corresponding plasmons are not spin-plasmons.

\section{Conclusions}

We set ourselves the task of studying the behavior of the dielectric screening function of bulk type electrons and plasmons in the inverted bands $\mathrm{HgTe}$ QWs at high temperatures and provide the comparison of the screening function for the Schrödinger fermions in this structure with the appropriate screening function for graphene monolayer with the Dirac fermions. The 
Random Phase Approximation dielectric screening function for the inverted bands $\mathrm{HgTe} \mathrm{QW}$, which takes into account realistic 8-bands k.p Hamiltonian for energy spectrum, was numerically simulated in this work.

It was found that for the case of static screening that has place for the electron scattering by charged impurities and holes, our dielectric function is similar to the graphene one in the long-wave limit. However, at the large values of scattering wave-vector $q$, the dielectric function for $\mathrm{HgTe}$ quantum well is several times smaller than the graphene one, which causes to the weaker damping of large-angle scattering by charged centers, and, as a result, leads to the lower mobility.

In the case of dynamical screening that describes the process of scattering by longitudinal optical phonons, there exists a maximum on the dependence of dielectric function on the scattering vector amplitude $q$, both for graphene and $\mathrm{HgTe}$ dielectric functions, for the fixed frequency. For the graphene case, this maximum grows to infinity. However, for $\mathrm{HgTe}$ dielectric function, this maximum is shifted to sufficiently lower values of $q$, while its height is finite, which affects the scattering by longitudinal optical phonons in the well.

Considering the dependence of screening function on the frequency at fixed values of wave-vector $q$, we can outline several important differences between graphene and HgTe QW screening functions. For graphene, there exists the sharp maximum on the plot; also there exists the minimum that is smoothed with increasing $q$. In the case of $\mathrm{HgTe} \mathrm{QW}$, this maximum of the screening function is strongly damped, forming the plateau. The peak value of this plateau is lower than that of the graphene plot; however the width of plateau is substantially broader than the width of peak in the graphene plot.

We also simulated the plasmons' frequencies of 2DEG in inverted HgTe QW in the long-wave limit for $T=77 \mathrm{~K}$. Plasmons in our case of HgTe QW are not the interface ones, because they are based on the 2D electron wave-function localized inside the well. As our RPA calculations are carried out for temperatures much greater than liquid helium temperature, appropriate plasmons are referred in this work as "high-temperature" plasmons. Plasmon frequencies in scattering vector dependences are represented as parallel straight lines in the doublelogarithmic scale for the HgTe QW and single-layer graphene.

Thus, all these peculiarities need to be taken into account when determining the response of these structures to external high frequency radiation, when creating detector structures.

\section{Acknowledgement}

This research was partly supported by VW Stiftung Project "Opto-electronic and transport phenomena in narrow gap semiconductor structures for terahertz detection" and NAS of Ukraine Program NANO №11/17-H.

\section{References}

1. Melezhik E.O., Gumenjuk-Sichevska J.V., Sizov F.F. Modeling of noise and resistance of semimetal $\mathrm{Hg}_{1-\mathrm{x}} \mathrm{Cd}_{\mathrm{x}} \mathrm{Te}$ quantum well used as a channel for $\mathrm{THz}$ hot-electron bolometer. Nanoscale Res. Lett. 2016. 11, No 1. P. 181.

2. Brüne C., Thienel C., Stuiber M., Böttcher J., Buhmann H., Novik E.G., Chao-Xing Liu, Hankiewicz E.M., and Molenkamp L.W. Diracscreening stabilized surface-state transport in a topological insulator. Phys. Rev. X. 2014. 4. P. 041045.

3. Hwang E.H., Das Sarma S. Dielectric function, screening, and plasmons in two-dimensional graphene. Phys. Rev. B. 2007. 75, No 20. P. 205418.

4. Ando T., Fowler A.B. and Stern F. Electronic properties of two-dimensional systems. Rev. Mod. Phys. 1982. 54. P. 437.

5. Melezhik E.O., Gumenjuk-Sichevska J.V. and Sizov F.F. Modeling of electron energy spectra and mobilities in semi-metallic $\mathrm{Hg}_{1-\mathrm{x}} \mathrm{Cd}_{\mathrm{x}} \mathrm{Te}$ quantum wells. J. Appl. Phys. 2015. 118, No 19. P. 194305.

6. Yi-Ping Lai, I-Tan Lin, Kuang-Hsiung Wu and JiaMing Liu, Plasmonics in topological insulators. Nanomaterials and Nanotechnology. 2014. 4, No 13. doi: 10.5772/58558.

7. Bouvier C., Meunier T., Kramer R., Levy L.P., Baudry X. and Ballet P. Strained HgTe: a textbook 3D topological insulator. arXiv, https://arxiv.org/abs/1112.2092v1 (2011).

8. Jin Li, Chaoyu He, Lijun Meng, Huaping Xiao, Chao Tang, Xiaolin Wei, Jinwoong Kim, Nicholas Kioussis, G. Malcolm Stocks \& Jianxin Zhong, Two-dimensional topological insulators with tunable band gaps: Single-layer $\mathrm{HgTe}$ and $\mathrm{HgSe}$. Sci. Repts. 2015. 5. P. 14115. doi: 10.1038/srep14115.

9. Danhong Huang, Zhitang Lin, Shixun Zhou, Dielectric response of a semi-infinite $\mathrm{HgTe} / \mathrm{CdTe}$ superlattice from its bulk anti surface plasmons. Phys. Rev. B. 1989. 40, No 3. P. 1672.

10. Dan-hong Huang, Jian-ping Peng and Shi-xun Zhou, Intrasubband and intersubband plasmons in a semi-infinite Fibonacci $\mathrm{HgTe} / \mathrm{CdTe}$ superlattice. Phys. Rev. B. 1989. 40, No 11. P. 7754.

11. Bansal M.L., Roy A.P., and Alka Ingale. Raman scattering from coupled plasmon-phonon modes in HgTe. Phys. Rev. B. 1990. 42, No 2. P. 1234.

12. Juergens S., Michetti P., and Trauzettel B. Screening properties and plasmons of $\mathrm{Hg}(\mathrm{Cd}) \mathrm{Te}$ quantum wells. Phys. Rev. B. 2014. 90. P. 115425.

13. Bernevig B.A., Hughes T.L., Zhang Shou-Cheng. Quantum spin Hall effect and topological phase 
transition in HgTe quantum wells. Science. 2006.

314. P. 1757-1761.

14. Meyer J.R., Arnold D.J., Hoffman C.A., Bartoli F.J. Electron and hole in-plane mobilities in $\mathrm{HgTe}-\mathrm{CdTe}$ superlattices. Phys. Rev. B. 1992. 46. P. 4139.

15. Nafidi A. Correlation Between Band Structure and Magneto-Transport Properties in $n$-type $\mathrm{HgTe} / \mathrm{CdTe}$ Two-Dimensional Nanostructure Superlattice. Application to Far-Infrared Detection, Chap. 6, in: Optoelectronics - Advanced Materials and Devices, Eds. S.L. Pyshkin and J.M. Ballato. InTech, 2013, p. 145. 